\title{
Hypothesis Scoring over Theta Grids Information in Parsing Chinese Sentences with Serial Verb Constructions
}

\author{
Koong H. C. Lin and Von-Wun Soo \\ Department of Computer Science, National Tsing-Hua University HsinChu, \\ Hsinchu, 30043, Taiwan, R.O.C. \\ E-Mail:soo@cs.nthu.edu.tw
}

\begin{abstract}
Serial verb constructions (SVCs) in Chinese are popular structural ambiguities which make parsing difficult. In this paper, we propose a quantitative model, 'S-model', based on theta grids information, that can systematically resolve ambiguities of SVCS to arbitrate competence between verbs in parsing SVCs sentences. S-model has three major characteristics: (I) it can resolve SVCS without relying on specific types of SVCs classified by linguists; (2) it can handle long $S V C s$, i.e., SVCs with more than two verbs; (3) it can simultaneously determine whether a verb candidate is really acts as a verb in the sentence.
\end{abstract}

\section{Introduction}

In Mandarin Chinese, it is common that there are two or more verbs in a sentence without any marker indicating the relationships between them. Such peculiar construct is called Scrial verb constructions (SVCs) [Li and Thompson 1981]. For example, in the sentence: "被 告希望原告諒解" (the defendant hope the plaintiff forgive) (The defendant hoped that the plaintiff could forgive him.), there are two verbs: "希望" (hopc) and "諒 解" (forgive); however, therc are no such markers as subordination markers, conjunctions, preposition, or other morphological cues, which indicate the relationships between them. In developing a parser, SVCs cause considerable problems. We have designed a modified chart parser using theta grids information. In parsing sentences with SVCs, different verbs will compete in searching the chart for their own theta rolcs. Thus, some mechanism for arbitrating among the competing verbs for the ownership of each constituent in the chart must be designed. The theta grid chart parser is to be described in the next section.

The study of SVCs is still primitive. Most previous work [Chang and Krulee 1991] [Yeh and Lee 1992] were based on Li and Thompson's classification of SVCs [Li and Thompson 1981]. Surveying their work, we find there are some limitations. Yang [1987] and Chang et al. [Chang and Krulee 1991] dealt with only subsets of SVCs. Moreover, it is not clear how the implementations of Yang [1987], Chang et al. [Chang and Krulce 1991], and Yeh et al. [Yeh and Lee 1992] can be extended to handle long SVCs, i.e., those sentences containing more than two occurrences of verbs. It is because thcir work were based on the classification of SVCs, and the classification was based on two-verbs cases only. Pun [1991] claimed that his work could handle long SVCs; however, did not report how to systematically extend his method to SVCs with three or more verbs. In our model, there are three characteristics: First, instead of classifying SVCs into several types, we makc use of a numerical scoring function to determine a prcferred structure. It is an attempt to make the SVCs handling process more systematic. The information encoded in theta grids are uscd as bascs for scoring. Second, it can handle long SVCS. Third, catcgory ambiguitics can be taken into consideration at the same time. Namely, we can simultaneously determine whether a verb candidate actually plays a verb or nol. While in previous work, before the SVC handling processes are triggered, it must determine the actual verbs in the sentence.

This work is part of our long-term research for building a natural language front-end of a verdict understanding system. Thus, the corpora we use are judicial verdict documents from the Kaohsiung district court [Taiwan 1990a][Taiwan 1990b], which werc written in a special official-document style. Thus, our analysis is based on such kind of sub-language.

\section{A Theta-grid Chart Parser}

Since the mechanism we propose is under the framework of a theta-grid chart parser, in this section, we introduce the parser briefly. Thematic information is one of the information sources that can bridge the gap between syntactic and semantic processing phases. In theta-grid theory [Tang 1992], rich thematic information is incorporated for the analysis of human languages. The idea of theta-grid theory is as follows: we use a predicate, say, a verb, as the center of a "grid" and, by finding the theta-roles registered in the lexical entries of this predicate, we can construct a grid formed by this predicate and then construe the sentence (or clause) spanned by this predicate. We think the theta-grid representation suitable for processing Chinese. This shares similar viewpoint with other work of designing Chinese parser which uses thematic information, such as ICG parser [Chen and Huang 1990]. To computationalize theta-grid theory, some control strategies for parsing must be implemented.

The well-known chart parser [Kay 1980], which utilizes the data structure "chart" to record the partial parsing results, is suitable for our work. Since it kceps all possible combination of constituents, it can accept sentences with missing theta roles. Thus, we designed a modified chart parser called TG-Chart parser [Lin and Soo 19931 by combining theta-grid theory and chart parser. Note that currently in our work, only the theta 
grids for verbs are considered. For each verb, there are two kinds of theta roles registered: the obligatory roles, which must be found for this verb to construct a legal "grid"; the optional roles, with their appcarance being optional. Take "告培" for example, its theta roles are registered as: + TTh (Pd) Agl; thus, two NPs must be found in the chart for the construction of a legal grid (From syntactic clues, both " $\mathrm{Ag}$ " and "Гh" are always played by NPs. [Liu and Soo 1993].), while the appearance of a clause to serve as a "Pd" role is optional A brict description of our parsing algorithm is as follows:

[Step 1] Search the sentence for positions of all "verb candidates". (What we call verb candidates are those words that have the verb-category as one of its syntactic categories in the dictionary.)

[Step 2] By considering all possible combination, the chart parser groups the words into syntactic constituents. Syntactic knowledge is used in this step.

[Step 3] If only one verb candidate is found in IStep 1], search the chart for constituents which can play the theta roles of this verb.

[Step 4] If more than one verb candidate are found, call $S$-model 10 determine the most preferred structure. Smodel will be described in section 3 .

\section{The S-model}

We design a model which utilizes scoring functions and theta-grid theory to handle the SVCs problem. This model, called S-model (an abbreviation of "SVCS handling model"), consists of four modules: a combination gencrator, a combination filter, a score cvaluator, and a structure selector as shown in [figure 1]. We now describe these modules as follows:

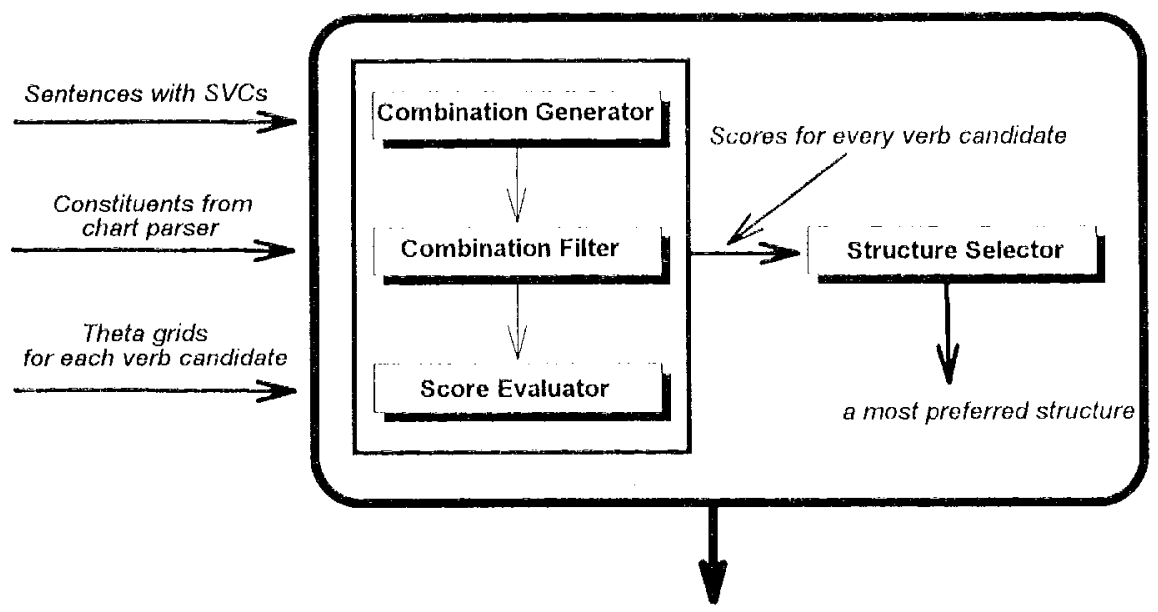

Return to TG-chart parser

Figure 1 Modules of S-model

As we know, all verb candidates compete to act as verbs. The question is: "which candidates can actually act as verbs?" and, "what is their correlation?". If we can enumerate all possible combination and evaluate their scorcs respectively, we can determine the most preferred construction. Take the two-verb-candidates case as an example, let the two verb candidates be $v 1, \mathrm{v} 2$, there are five combination: (1) only $v 1$ is a verb while v2 is not, (2) only $\mathrm{v} 2$ is a verb, (3) both $\mathrm{v} 1$ and $\mathrm{v} 2$ are verbs, while there is not any subordination relation between them. (4) both are verbs, and $v 1$ is subordinate to $v 2$. (5) both are verbs, and $\mathrm{v} 2$ is subordinate to $\mathrm{v} 1$.

\subsection{Combination Generator}

Combination Gencrator consists of two submodules: Verb-string Generator and Subordination-relation Tagger. We illustrate a case with three verb candidates
Verb-string Gencrator gencrates all possible verb strings by sequentially conumerating the binary string: 001,010 $011,100,101,110,111$. The verb string "101" represents the situation where vl and v3 act as verbs, while $v 2$ doesn't. And then, Subordination-relation 'Tagger tags these verb strings with possible subordination relations. It divides these strings into three classes according to the occurrences of $I$ 's in the string, that is, the number of verb candidates in the sentence. These three classes are: (1) For the one-1 class (i.e., 001 , $010,100)$, there is obviously no subordination relation. That is, there is only one possible case to consider: this candidate acts as the only verb in this sentence. (2) for the two-1 class (i.e., $011,101,110$ ), there are three possibilities to consider: $v 1=\mathbf{v} 2, \mathbf{v} 1<\mathbf{v} 2$, and $\mathbf{v} 1>\mathbf{v} 2$. WC follow the nolations used by Pun [Pun 1991], where "v1>v2" mcans $\mathrm{v} 2$ is subordinate to $\mathrm{vl}$; " $\mathrm{v} 1=\mathrm{v} 2$ ", no subordination relations exist between the two verbs. (3) 
For the three-1 class (i.e., 111), there are seventeen cases. We use abbreviated notations to represent them, where " $><$ " is the abbreviation of "vl $>[v 2<v 3]$ ", with square brackets being represented by underlines, meaning that locally $\mathrm{v} 2$ is subordinate to $\mathrm{v} 3$, and they together form a clause, which then plays a prepositional role for $\mathrm{vl}$, and, for another example, " $\equiv<"$ is the abbreviation of " $[\mathrm{v} 1=\mathrm{v} 2]$ $<\mathrm{v} 3^{\prime \prime}$. These seventeen cases arc: $==,=<,=<,=>, \Rightarrow \geq$, $<=,<=, \leq<,<<,>,<,>=,>=, \geq<,>\leq, \geq>$, and $>>$ These cases are generated simply by cnumerating possible combinations of these three symbols: $=<$, and > For each pair of symbols $S_{1} S_{2}$, two combinations are possible: $S_{1} S_{2}$ and $S_{1} S_{2}$. Note that "==" and "==" represents the same case; thus, only a single " $=="$ is generated. Therefore, $3 \times 3 \times 2-1=17$ cases arc possible. By summarizing classes (1), (2), and (3), Combination Gencrator generates $C_{1}^{3} \times 1+C_{2}^{3} \times 3+C_{3}^{3} \times 17=29$ cases. It is easy to design a routine which systematically enumcrates these possibilitics.

\subsection{Combination Filter}

The Combination Generator above docs not take linguistic knowledge into consideration. Actually, there are some cases which will never happen in a real sentence according to syntactic constraints. Thus, it is not necessary to pass it to the score evaluator Combination Filter is responsible for filtering out impossible cases. We illustrate three circumstances. Firstly, for "v1 > v2", the Combination Filter will check the theta grid for $v 1$; if there is a $\mathrm{Pd}$ or Pe role registered in vl, it is possible, since $\mathrm{v} 2$ can be subordinate to $\mathrm{vl}$ only if $\mathrm{v} 1$ also expects a prepositional role; otherwise, such a case is filtered out. The second circumstance is, when $v 1$ has only a single syntactic category, verb, it must act as a verb in the sentence. Thus, the case that v2 acts as a verb while vl docsn't is removed. The third circumstance regards the three-candidates situations. Combination Generator generates seventeen cases; however, under some circumstances, there are four cases which are impossible: $<<,<, \leq>$, and $\gg$. These circumstances happens when the main verb of the prepositional part (i.e., the part marked by a underline.) expects an animate agent. In such circumstances, a VP cannot be subordinate to an "event". Thus, these four will be filtered out by Combination Filter. For example, the following sentence, with the relation " $\$$ " (i.c., 打 $<$

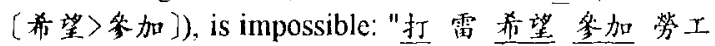
保 " (play thunder hope attend the labor insurance)
(Thundering hoped to attend the labor insurance.). It is because "弟望" expects an animate NP to act as its $\mathrm{Ag}$, the VP "打雷" thus cannot act as its $A g$ role.

There are still many linguistic knowledgc and constraints which can be used by Combination Filter. However, some of them, such as the third circumstance mentioned above, are too specific and thus must be used carcfully to avoid over-constraints. Therefore, how to collect and select those constraints and knowledge which are general enough is still our future concern.

The main function of Combination Filter is to improve the performance of the S-model. Note that in this paper, for the beneficiary of brevity, Combination Generator and Combination Filter are designed as two scparate modules. However, Combination Filter can behave as an embedded module of Combination Gencrator so that it can cut off some generating branches which arc impossible as carly as possible. It is also our future concern.

\subsection{Score Evaluator}

Whenever Combination Filter passes a feasible case into Score Evaluator, the Score Evaluator utilizes a scoring function to compute the score of the input case and then, passes the evaluated score to the structure selector. We will now describe it:

\subsubsection{The S-function}

In our legal domain corpora, there are many occurrences of SVCs. Since our parser is based on the theta grids, in case of SVCs, different verbs will compete in finding their own theta roles. Thus, some mechanism for arbitrating among verbs for the ownership of each constituent in the chart must be designed. Just as what Yorick Wilks said, language does not always allow the formation of "100\%-correct" theories [Hirst 1981]; therefore, we attempt to find a more flexible method for recognizing SVCs. We propose a scoring function to select a "preferable" construction for the sentence with SVCs. (For the "preference" notion, sec [Wilks 1975] [Fass and Wilks 1983].) The scoring function is called Sfunction, an abbreviation for "SVCs scoring function" S-function is defined as in [figure 21, where RWR is the abbreviation of "Ratio of Words included in some phrase with Roles assigned", RRF, "Ratio of Roles Found", OBR, "OBligatory Rolc", and OPR, "OPtional Rolc" (Note that OBR and OPR indicate those roles registered in theta grids.): 
(3) "請求 " and "蜼婚" both are treated as verbs. Score $=(0.134+0.67) / 2=0.402$

(4) "請求" and "離婚" both are treated as verbs, with " 岞婚" being subordinate to "請求"

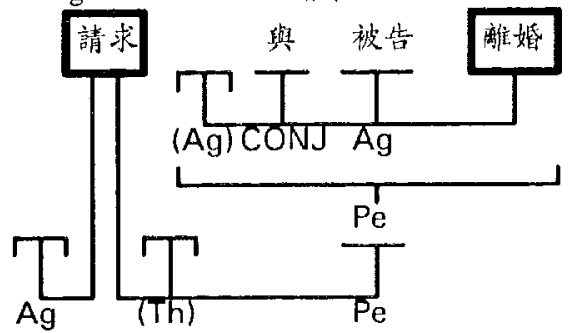

For "請求", Base $=5$. RWR $=4 / 4=1$. Score-Per-Verb $=$ $\{[1 * 2+0] / 5\}=0.4$. For " 離 婚 ", Base $=3$. $\mathrm{RWR}=3 / 3=1$. Score-Per-Verb $=\{[1 * 2+0] / 3\}=0.67$. Score $=(0.4+0.67) / 2=0.535$.

(5) "請求" and "㒕婚" both are treated as verbs, with " 請求" being subordinate to "離婚". Score= $(0.134+0) / 2=0.067$.

From the above discussions, case(4) apparently gets the highest score (0.535). So, the parsed structure in case(4) is preferable to those in the other cases. That is, in this sentence, "請求" and "雖婚" both are treated as verbs, while "離婚" is subordinate to "請求". The clause constructed by "離婚" is assigned the Pe role for "請求". Thus, this is a SVC sentence; moreover, this kind of SVC is commonly called "sentential objects".

\subsection{Structure Selector}

Structure Sclector plays a final arbitrator. It collects all feasible cases and their scores. After scorcs of all cases are evaluated, the competition of all cases is arbitrated by Structure Selector. Structure Selector selects the case with the highest score as the most preferred one. The final result is returned to the parser.

\section{Experimental Results}

\subsection{Results of More Sample Sentences}

In table 2, we show the results of more sentences with $\mathrm{SVC}$ in the legal documents which are parsed by this scheme in our TG-Chart parser. The sample sentences are shown in table 1:

Table 1. Some sample sentences with SVCs

S1:原告 訴請 被告 給予 三十萬元 (the plaintiff petition the defendant give three hundred thousand dollars)

The plaintiff petitioned the defendant to give him three hundred thousand dollars.

\$2: 原告 請求 被告 清龦 债務 (the plaintiff request the defendant repay dehts)

The ptaintiff requested the defendant to repay his debts.

S3: 被告末 到場爭執 (he defendant didn't anive the court argue)

The defendant didn't arrive at the court to argue.

S4: 被告 突 無故 離家 出走 (the defendant suddenly causelessly left home desert his family)

The defendant deserted his family suddenly and causelessly.

S5: 被告 未渥家與原告 同居 (the defendant didn't return home with the plaintiff cohabit)

The defendant didn't retum home to cohahit with the plaintiff.

S6: 原告 請訊問 證人 (the defendant petition interrogate the witness)

The defendant petitioned to interrogate the witness.

57: 被告 希望 原告 能 諒解 (the defendant hope the plaintiff can forgive)

The defendant hoped that the plaintiff could forgive him.

S8: 被告 申請 參加 勞工保險 (the defendant apply attend the labor insurance)

The defendant applied to attend the labor insurance.

s9: 原告平時待人很和睦（the plaintiff ordinarily treat people very friendly)

Ordinarily, the plaintiff treats people friendly.

s10: 原告打破 3 一個花瓶很值錢 (1he plaintiff break Asp one vase very valuable)

The plaintiff broke a vase that was valuable.

Table 2. Results of S-function calculation for sample sentences

\begin{tabular}{|c|c|c|c|c|}
\hline 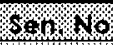 & 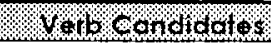 & 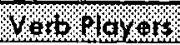 & 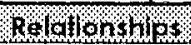 & 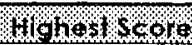 \\
\hline $151 \%$ & $\mathrm{v1}$ : 彭請, $\mathrm{v} 2$ : 給予 & $\mathrm{v} 1, \mathrm{v} 2$ & $\mathrm{v} 1>\mathrm{v} 2$ & 1.00 \\
\hline S2. & v1: 請求,v2: 清償 & $\mathrm{v} 1, \mathrm{v} 2$ & $\mathrm{v} 1>\mathrm{v} 2$ & 1.00 \\
\hline 8 & $\mathrm{v1}$ : 到, v2: 爭乹 & $\mathrm{v} 1, \mathrm{v} 2$ & $\mathrm{v} 1=\mathrm{v} 2$ & 0.84 \\
\hline s4. & $\mathrm{vl}$ : 離家, v2: 出走 & $\mathrm{v1}, \mathrm{v} 2$ & $\mathrm{v} 1=\mathrm{v} 2$ & 1.00 \\
\hline 8 & $\mathrm{v} 1$ : 返， v2: 同居 & $\mathrm{v} 1, \mathrm{v} 2$ & $\mathrm{v} 1=\mathrm{v} 2$ & 0.83 \\
\hline 86 & v1: 馨請, v2: 訊問 & $\mathrm{v1}, \mathrm{v} 2$ & $v 1 \geq v 2$ & 0.70 \\
\hline S/ & v1: 希望,v2: 諒解 & $\mathrm{v} 1, \mathrm{v} 2$ & $\mathrm{v} 1>\mathrm{v} 2$ & 0.84 \\
\hline 83 & v1: 申請,v2:參加 & $\mathrm{vl}, \mathrm{v} 2$ & $\mathrm{v} 1>\mathrm{v} 2$ & 0.75 \\
\hline 80 & $\mathrm{v} 1$ : 待, $\mathrm{v} 2$ : 和睦 & $\mathrm{vl}, \mathrm{v} 2$ & $\mathrm{v} 1<\mathrm{v} 2$ & 1.00 \\
\hline$\$ 10$ & $\mathrm{v1}$ ：打破，v2：値䤦 & $\mathrm{vl}, \mathrm{v} 2$ & $\mathrm{vl}=\mathrm{v} 2$ & 1.00 \\
\hline
\end{tabular}




\subsection{Demonstrating How to IIandle Three-Verbs SVCs}

Let's consider the following three-verbs sentence: "

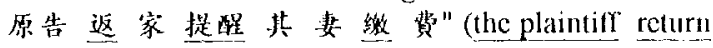
home remind his wife pay fees) (The plaintiff returned home to remind his wife to pay fees.). "There are three verbs in this sentence: 这 (return), 提理 (remind), and (pay). At the first stage, Combination Generator generates 29 possible combination; and then, Combination Filter filters out 26 of them, and only three cases remaincd to be considered: "返 =提醒 = 緅", "返 =

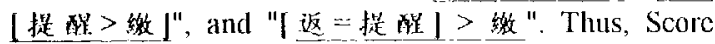
Evaluator only needs to calculate the scores for these three remained cascs. At the final stage, Structure Selector accepts the evaluated scores for these cases and selects the one with the highest score. In this example, the structure $" \Rightarrow$ " gets the highest score: 0.94 ; it is the correct structure for this sentence.

Consider another interesting cxample, "他以为我 嘲笑他是鍇的" (he think I mock he is wrong) [Pun 1991]. This sentence is ambiguous to native speakers, since there are two possible readings: (1) "I 他 以為我嘲笑他了是错的" (His thinking that I mocked him is wrong.), and (2) "他以为 [我嘲笑他是錯的]" (He thinks that I mocked him for being wrong.). In Smodel, both these two readings get the highest score: 1.0 , and thus both are selected by Structure Sclector as the linal output. S-model doesn't attempt to select a "uniquely-correct" structure, but just selects what are preferred. It matches humans' behavior since cven a human may not be able to tell which of these two is better.

\section{Conclusion}

In this paper, we propose a systematic method for analyzing SVCs. The method is based on the information offered by theta grids. Many possible correlation relations may exist between verbs, we use a numerical scoring function to determine the most preferred one. To utilize the S-function defined, we design a S-model, which consists of four modules: a combination generator, a combination filter, a scorc cvaluator, and a structure selector, to realize it. For the examples we have tested so far, taken from the legal documents [Taiwan90a| |Taiwan90b|, our mechanism always produces the correct reading.

$\mathrm{Li}$ and Thompson [1981] classified SVCs into four types: (1) two or more separate events (2) a VP or a clause plays the subject or direct object of another verb (3) pivotal construction (4) descriptive clauses. We usually split type (2) into two sub-types: (2)-1 sentential subjects, and (2)-2 sentential objects. Most work for handling SVCs arc bascd on this classification. In our design of Sfunction, information about this classification is not used However, in our testing sentences, it turns out that these five types are actually covered by the S-model which selects a preferred structure based on only scoring functions. For example, S5 in table 1 belongs to type (1),
S9, type (2)-1, S6, type (2)-2, S2, type (3), and S10, type (4). The reason why S-model may cover the classification is due to the rich information encoded in theta grids. As an example, consider the sentence "被告 整請訉间證人". (The defendant petitioned to interrogate the witness.) By $\mathrm{Li}$ and Thompson's classification, it belongs to the "sentential objects" type. If we can classify the sentence into the correct type, the

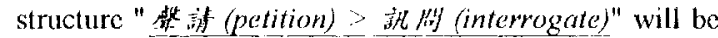
determined. This is the idea used in most previous work However, in S-model, we achieve the same result without relying on the classification. In S-model, since " 呫部" needs a "Pe" which implies that it expects an "cvent", i.c., a "sentential object" to play the thela role, after calculating S-function, the structure where " subordinate to "解就" naturally gets the highest score and thus becomes the "winner". As the previous cxample in section 4.2, for the ambiguous sentence S-model also yields more than one highest scorc. We can conchude that S-model could be a very general and sound mechanism to handle SVC sentences.

\section{Acknowledgment}

This rescarch is supported in part by National Science Council of R.O.C. under the grant NSC8.3-0408-E-007008

\section{References}

[Chang and Krulee 1991] Chao-Huang Chang and Gilbert K. Krulce, Prediction Ambiguity in Chinese and Its Resolution. Proc. of ICCPCOL 1991, pp, 109-114

[Chen and Huang 1990] Kch-jiam Chen and Chu-Ren Huang, Information-based Case Grammar. In Proc. of COLING-90

[Fass and Wilks 1983] Dan Fass and Yorick Wilks, Preference Semantics, Ill-Formedness, and Metaphor. American Journal of Computational Linguistics, Vol. 9 (3-4), July-December 1983, pp. 178-187.

[Gruber J. S. 1976] Gruber J. S., Lexical Structures in Symtax and Semantics, North-Holland Publishing Company. 1976.

[Hirst 1981] In Graenc Hirst, Lecture Notes in Computer Science, Anaphora in Natural Language Understanding, A Survey. Springer-Veriag Berlin Heidelberg. 1981 .

[Kay 1980] Martin Kay. Algorithm Schemata and Data Structures in Syntactic Processing. In Proc. of the Nobel Symposium on Text Processing, Gothenburg, 1980.

[Li and Thompson 1981] C. N. Li and S. Thompson, Mandarin Chinese: a liunctional Reference (rrammar, University of California Press, Berkeley. 1981.

[Lin and Soo 1993] Koong H.C. Lin and Von-Wun Soo, Toward Discourse-guided Chart Parsing for Mandarin Chinese -.. A Preliminary Report. ROCLING VI, 1993

[Liu and Soo 1993] Rey-Long Liu and Von-Wun Soo, An timpirical study of Thematic Knowledge Acquisition Based on Syntactic Clues and teuristics. In Procecdings of $\mathrm{ACl}, 1993$. 
[Pun 1991] K. H. Pun, Analysis of Serial Verb Constructions in Chinese. ICCPCOL 1991, pp.170-175. 1991.

[Taiwan 1990a] Taiwan Kaohsiung district court, Summary of Kaohsiung District Court Criminal Verdict Documents, Vol. 1, 1990.

[Taiwan 1990b] Taiwan Kaohsiung district court, Summary of Kaohsiung District Court Civil Verdict Documents, Vol. 1, 1990.

[Tang 1992] Ting-Chi Tang, Syntax Theory and Machine Translation: Principle and Parameter Theory. In Proc. of ROCLING V, pp.53-83. 1992.

[Yang 1987] Yiming Yang, Combining Prediction, Syntactic Analysis and Semantic Analysis in Chinese Sentence Analysis. IJCAI 1987, pp.679-681.

[Yeh and Lee 1992] Ching-Long Ych and Hsi-Jian Lee, A Lexicon-Driven Analysis of Chinese Serial Verb Constructions. In Proc. of ROCLING V, pp.195-214. 1992.

[Wilks 1975] Yorick Wilks, An Intelligent Analyzer and Understander of English. In B. J. Grosz, K. S. Jones, and B. L. Webber, "Reading in Natural Language Processing", 1975. 